\title{
Villous left atrial myoxma-a rare case report
}

\section{Abstract}

64 year old female who presented with dyspnea for two months duration was evaluated in our department. Echocardiogram showed a cauliflower like mass with multiple protruding villous appearance attached to the inter atrial septum near the foramen ovale suggestive of left atrial myxoma traversing the mitral valve orifice causing mitral valve obstruction. Patient was referral to cardiothoracic surgeon for surgical excision.

\author{
Volume II Issue 6 - 2018
}

\section{Thangaraju A, Ravi MS, Swaminathan N,} Venkatesan S, Cecily Mary Majella J

Institute of Cardiology, Madras Medical College \& Hospital

Correspondence: Swaminathan N, Institute of Cardiology, Madras Medical College \& Hospital, India, Email dr.msesi2008@gmail.com

Received: January 28, 2017 | Published: December 13, 2018

\section{Introduction}

The prevalence of cardiac tumours at autopsy ranges from $0.001 \%$ to $0.3 \%$ of which more than $50 \%$ are benign. Myxomas are the most common primary tumor of the heart. Typical myxomas are pedunlucated and gelatinous in consistency. Tumors vary in size from 1-15cms; weighing, 15-180 g-n-? 35\% of myxomas are villous and friable with increased tendency for embolization. Large tumours are more likely to be associated with cardiovascular symptoms. Intelieukin-6 synthesized by the myxoma is responsible for the constitutional symptoms.

\section{Case report}

We present a 64 _ year old female who presented with symptoms of dyspnea NYHA class III, and palpitations for two months duration. Clinical examination revealed pallor, and a mid diastolic murmur in mitral area. ECG was within normal limits. Echocardiogram showed a cauliflower like mass with multiple protruding villous appearance attached to the inter atrial septum near the foramen ovale traversing the mitral valve orifice causing mitral valve obstruction. Patient was referred to cardiothoracic surgeon for surgical excision (Figure 1,2).

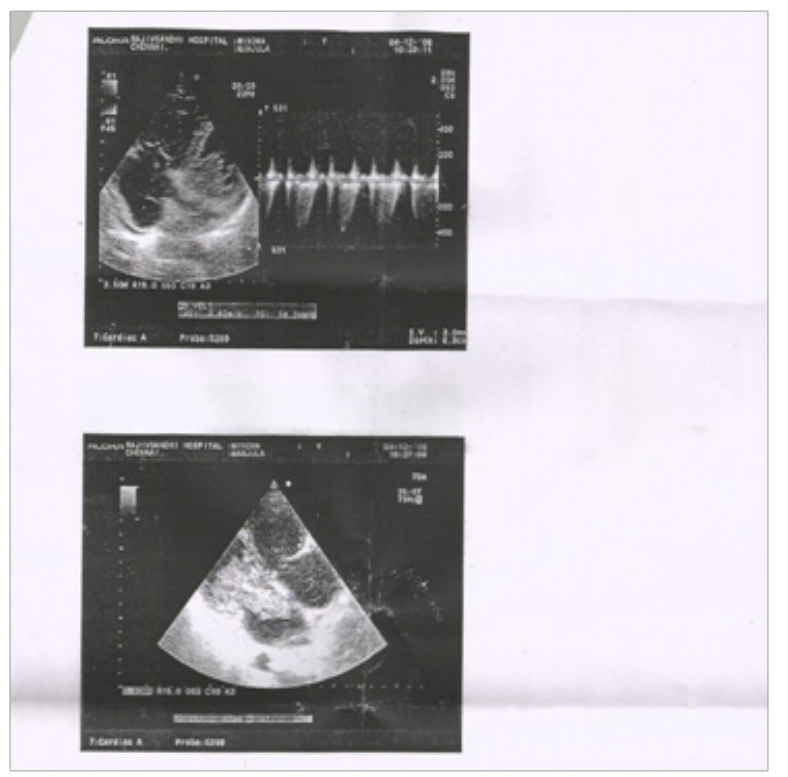

Figure I Clinical examination I.

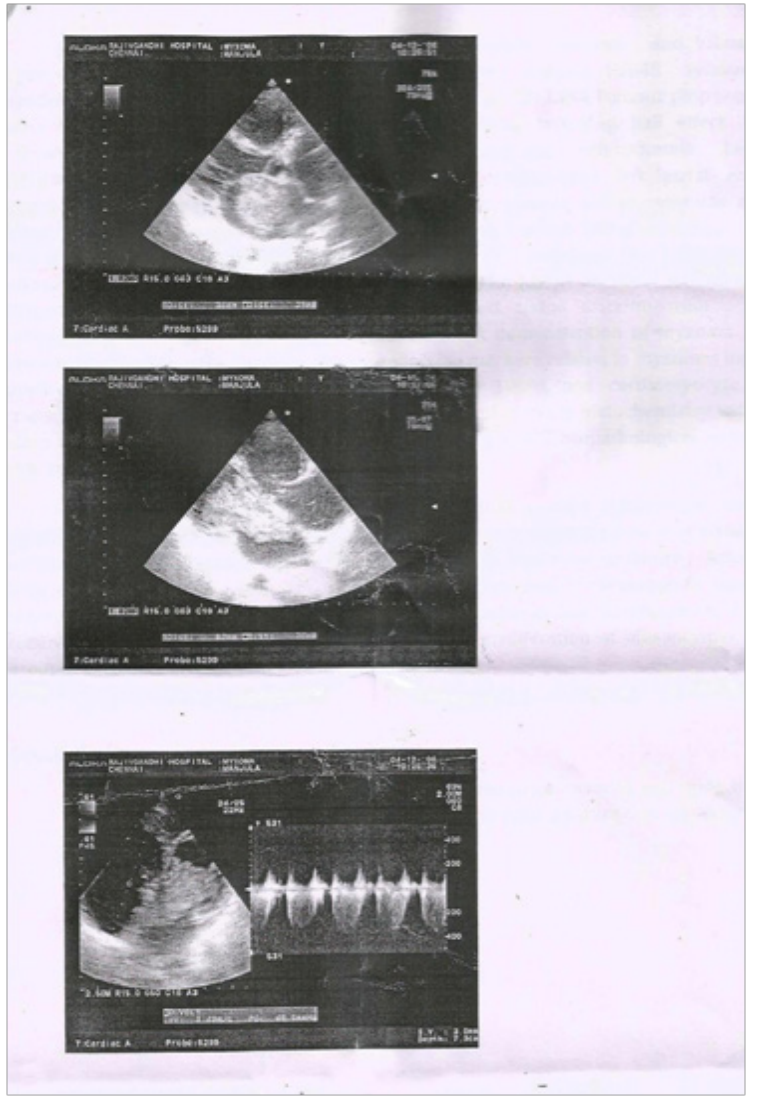

Figure 2 Clinical examination 2.

\section{Discussion}

Atrial Myxoma being the most common primary cardiac tumour ${ }^{1}$ is usually solitary [90\%] and presents more frequenitly in women. Symptoms depend upon the site, size and mobility of the tumour. Most common site is the left atrium. An extensive literature review has been performed to present comprehensive review of the cause and pathophysiology of atrial myxoma. Clinical manifestations include triad $^{2}$ of Constitutional symptoms.

\section{Obstructive symptoms and embolic manifestations}

Constitutional symptoms ${ }^{3}$ include fever, weight loss, myalgia, rash, arthralgia, clubbing, weakness , and raynauds phenomenon. 
Obstructive symptoms comprises of exertional dyspnea, paroxysmal nocturnal d-yspn-ea, syncope, and even sudden death. Embolic manifestations comprises of stroke, seizures, and infarctions involving cerebellum, brainstem, spinal cord, and retina.It masquerades as mitral stcnosis. Myxoma is classified as familial myxoma, sporadic myxoma, and villous myxoma Syndromes associated with myxomas include Carney's complex, NAME syndrome, LAMB syndrome. Cardiovascular examination reveals Loud S1,LVS3+,LVS4, Tumour plop sound -100ms after S2, Mid diastolic murmur due to - obstructionwrecking ball effect. Laboratory investigations reveal elevated' ESR, Thrombocytosis, Polycythemia, Leukocytosis, Anaemia, increased IL-6.X-Ray Chest may reveal cardiomegaly left atrial enlargement, calcification. ECG may show -left atrial enlargement, various tachyarrythmias and heart block. Echo classification ${ }^{4}$-Class I Small and prolapsing through mitral valve, Class II Small and non prolapsing, Class III -Large and prolapsing, Class IV - Large and Non prolapsing. 2D Echo adequate to diagnose myxoma with $95 \%$ sensitivity. TEE 5,6 is the most sensitive method to diagnose myxoma. CT Scan: may demonstrate soft tissue discrimination, myocardial infilteration and Calcification. MRI: reveals excellent demonstration of myxoma. Angiogram shows filling defect in the chamber. Neuro endocrine markers related to myxomas include S100 positivity rate $89 \%$, Cal Retinin -positivity rate $100 \%$, and cardiomyocyte - specific transcription factors. Biopsy: Fine needle aspiration and immuno histochemistry with Vimentin stain and for Annexin V2 is valuable in the diagnosis. Histopathological examination is charateristic of Lipid cells.

Recurrence of cardiac myxoma is due to familial myxoma. Recurrence rate $1-3 \%$ for sporadic myxoma and $10-20 \%$ for familial myxomas.Conventional treatment of atrial myxoma is surgical removal. Complete operative resection is the treatment of choice. Annuloplasty is indicated for damaged valves. Prosthetic valve replacement is necessary in case of severe obstruction and destruction of the valve. Cardiac transplantation recommended for multiple, recurrent atrial myxoma. Myxoma cause excessive proliferation of mesencymal cells which produce excessive glycosaminoglycan production.

\section{Conclusion}

This case of villous left atria ${ }^{1}$ myxoma is presented for its rarity and since it remained asymptomatic till the age 64 inspite of its virulent villous nature and tendency to embolise.

\section{Acknowledgments}

None.

\section{Conflict of interest}

Author declares that there is no conflicts of interest.

\section{References}

1. Reynan K. Frequency of the primary Tumours of Heart. Am J Cardiol. 1996;77(1):107.

2. Reynan K. Cardiac Myxoma. N Engl J Med. 1996;333(24);1610-1617.

3. Talley DJ, Wenger NK. Atrial Myxoma: Overview, Recognition, and Managements. Compr Ther. 1987;13(4):12-18.

4. Charuzi Y, Bolger A, Beeder C, et al. A New Echocardiographic classification of Left Atrial Myxoma. Am J Cardiol. 1985;55(5):614-615.

5. Finogan RE, Harrison DC. diagnosis of Left Atrial Myxoma by Echocardiography. N Engl J Med. 1970;282(18):1022-1023.

6. Spencer WH, Peter RH, Orgain ES. Detection of Left Atrial Myxoma by echocardiography. Arch Intern Med. 1971;128(5):787-789. 